\title{
Impact of Gain Saturation on the Parametric Amplification of 16-QAM Signals
}

\author{
Da Ros, Francesco; Borkowski, Robert; Zibar, Darko; Peucheret, Christophe
}

Published in:

ECOC Technical Digest

Link to article, DOI:

10.1364/ECEOC.2012.We.2.A.3

Publication date:

2012

Document Version

Publisher's PDF, also known as Version of record

Link back to DTU Orbit

Citation (APA):

Da Ros, F., Borkowski, R., Zibar, D., \& Peucheret, C. (2012). Impact of Gain Saturation on the Parametric Amplification of 16-QAM Signals. In ECOC Technical Digest (pp. We.2.A.2). Optical Society of America. https://doi.org/10.1364/ECEOC.2012.We.2.A.3

\section{General rights}

Copyright and moral rights for the publications made accessible in the public portal are retained by the authors and/or other copyright owners and it is a condition of accessing publications that users recognise and abide by the legal requirements associated with these rights.

- Users may download and print one copy of any publication from the public portal for the purpose of private study or research.

- You may not further distribute the material or use it for any profit-making activity or commercial gain

- You may freely distribute the URL identifying the publication in the public portal 


\title{
Impact of Gain Saturation on the Parametric Amplification of 16-QAM Signals
}

\author{
Francesco Da Ros, Robert Borkowski, Darko Zibar and Christophe Peucheret
}

Department of Photonics Engineering, Technical University of Denmark, DK-2800 Kgs. Lyngby, Denmark, $\bowtie$ fdro@fotonik.dtu.dk

\begin{abstract}
The effect of gain saturation on parametric amplification of 16-QAM signals is investigated in terms of signal distortion. The relative impact of gain saturation, nonlinear phase rotation and nonlinear phase noise is discussed. Experimental results at $14 \mathrm{GBd}$ confirm the conclusions of the numerical analysis.
\end{abstract}

\section{Introduction}

The continuously increasing demand for capacity has resulted in intensive investigations of spectrally efficient multilevel modulation formats. Among those, 16-quadrature-amplitudemodulation (16-QAM) is of great interest, allowing a high spectral efficiency as well as a fourfold increase in the bit rate for a given symbol rate and the ability to exploit the advances in digital coherent receivers ${ }^{1}$. Further capacity increases may be provided by expanding the current optical communication transmission bands. Fiber optical parametric amplifiers (FOPAs) represent a concrete opportunity to move towards this direction, combining the ability to provide a large flat gain with a broad amplification bandwidth at arbitrary wavelengths. Gain bandwidths of $100 \mathrm{~nm}$ have been demonstrated in single pump FOPAs ${ }^{2}$.

Investigations on the use of parametric amplification for multilevel modulation formats have only been initiated very recently. The bit-error-ratio (BER) performance of parametrically-amplified 16-QAM signals has been theoretically analyzed ${ }^{3}$ and experimentally demonstrated within the linear regime of the FOPA ${ }^{4}$. However, since FOPAs rely on the ultra-fast Kerr nonlinearity in highly nonlinear fibers (HNLFs), the effect of gain saturation will result in a symbol-dependent gain, hence in distortions of the 16-QAM constellation. This effect has not been systematically analyzed so far.

In this contribution we investigate numerically and experimentally the impact of gain saturation on the amplification of 16-QAM signals. The error vector magnitude (EVM) metric is used to quantify the distortion since, by enabling a segregation between symbols, it provides a better insight than the BER on the physical phenomena causing the signal degradation. In particular, we analyze the concurrent effects of gain saturation and nonlinear phase rotation. An experimental investigation at $14 \mathrm{GBd}$ confirms the numerical findings.

\section{System under investigation}

The system investigated is represented in Fig. 1.
A 16-QAM signal obtained from two 4-pulse amplitude modulation (4-PAM) driving signals in a conventional in-phase and quadrature (IQ) modulator is coupled together with a continuous wave (CW) pump into the HNLF, implementing a single pump FOPA. At the output of the HNLF, the amplified signal is selected with an optical bandpass filter (OBPF) and received using a digital coherent receiver. Offline signal processing is used in the experiment.

The signal and pump wavelengths are set to $1550.0 \mathrm{~nm}$ and $1557.5 \mathrm{~nm}$, respectively. The pump power at the HNLF input is fixed at $28 \mathrm{dBm}$ while the signal power is swept to investigate the effect of gain saturation. The HNLF length, zerodispersion wavelength, dispersion slope, nonlinear coefficient and attenuation are $500 \mathrm{~m}$, $1550.4 \mathrm{~nm}, 0.0185 \mathrm{ps} /\left(\mathrm{nm}^{2} \cdot \mathrm{km}\right), 10.7 \mathrm{~W}^{-1} \cdot \mathrm{km}^{-1}$ and $0.7 \mathrm{~dB} / \mathrm{km}$, respectively. The inset of Fig. 1 shows simulated ON-OFF gain spectra for a CW signal with various input power levels, clearly showing the impact of saturation when the signal input power is increased from -20 to $0 \mathrm{dBm}$.

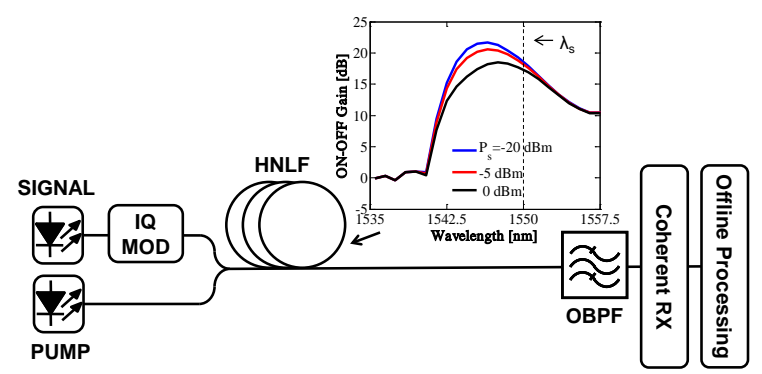

Fig. 1: System setup. The inset shows the simulated ON-OFF gain spectra for various signal power levels at the input of the HNLF.

\section{Numerical analysis}

Numerical simulations have been carried out by solving the nonlinear Schrödinger equation describing the wave propagation in the HNLF by the split-step Fourier method. Furthermore, the coherent receiver has been assumed ideal with 
carrier recovery implemented using the Viterbi \& Viterbi algorithm with QPSK partitioning ${ }^{5}$. Finally, the EVM has been calculated as the root-meansquare error between the received symbol $S_{i}$ and the ideal symbol $\mathrm{S}_{0, k}$,

$$
\mathrm{EVM}_{k}=\sqrt{\frac{\frac{1}{N} \sum_{i=1}^{N}\left|S_{i}-S_{0, k}\right|^{2}}{\max _{l}\left|S_{0, l}\right|^{2}}},
$$

choosing a data-aided approach ${ }^{6}$ in order to evaluate accurately the signal distortion. The EVM has been calculated separately for the sixteen constellation clusters according to (1). A "cluster" is defined as the set of received points corresponding to the transmission of the same modulation state among the sixteen possible in the QAM constellation. This approach allows to resolve the symbol-dependent effects.

The analysis is performed first with a noise-free 16-QAM signal. The EVM is shown in Fig. 2(a) as a function of the average signal power at the HNLF input for baud rates $\left(R_{s}\right)$ of 14 and $28 \mathrm{GBd}$. The sixteen curves, one for each cluster, are actually split into three groups depending on the power of the transmitted symbol in the 16-QAM constellation. These groups will be referred to as outer, middle and inner clusters, depending on their power levels, and color coded as shown in the inset. The EVM performance of quadrature phase-shift keying (QPSK) modulation is also reported as a reference.

It can be noticed that the EVM performances are not baud rate dependent since the parametric gain is an ultra-fast process. Regardless of the cluster and the modulation format, the EVM increases with increasing input power, however at different rates. The distortion is the strongest for the outer clusters, as expected, since they carry the highest power. However, the second most distorted clusters turn out to be the inner ones. It is essential to remember at this point that, due to the ultra-fast nature of the Kerr nonlinearity, the saturation is caused by the instantaneous power carried by each symbol, and not by the average power. Fig. 2(b) shows the signal gain for the three sets of clusters. The gain experienced by each symbol can be directly related to its power, thus the outer clusters saturate first and the inner clusters last. The middle clusters show the same performance as a QPSK signal since they carry the same power.

In the saturation regime, the outer clusters experience a lower gain. Therefore, they are being shifted towards a lower power level in the output constellation, compared to an ideal constellation with the same average power. Simultaneously the inner clusters are only slightly affected by the saturation and thus are relatively shifted towards higher power. The combination of these effects results in an amplitude squeezing of the constellation as the gain difference reaches almost $6 \mathrm{~dB}$ for $P_{s}=0 \mathrm{dBm}$. The EVM for both sets of clusters grows since they are shifted away from their original position in an ideal constellation. The lowest EVM increase is therefore experienced by the clusters the least affected by this power limiting effect, i.e. the middle ones, as their gain curve is in-between outer and inner clusters.

Finally, the EVM trend for the middle clusters reaches a stable value at around $-3 \mathrm{dBm}$ of input power. The second power-dependent effect the clusters are affected by, namely nonlinear phase rotation, needs to be taken into account to explain this observation. The cluster-dependent power, together with the gain difference, results in a relative nonlinear phase shift between the three power levels. In the specific case of the middle clusters in saturation, even though the spreading of the cluster points increases with the power, the phase rotation is dominant and does not increase so significantly, thus pinning the EVM down to a constant value.

The EVM performance was further analyzed with a noisy signal at the input of the FOPA. Complex white Gaussian noise was added to the signal according to a given optical signal-to-noise ratio (OSNR) specified in the usual $12.5 \mathrm{GHz}$ bandwidth.
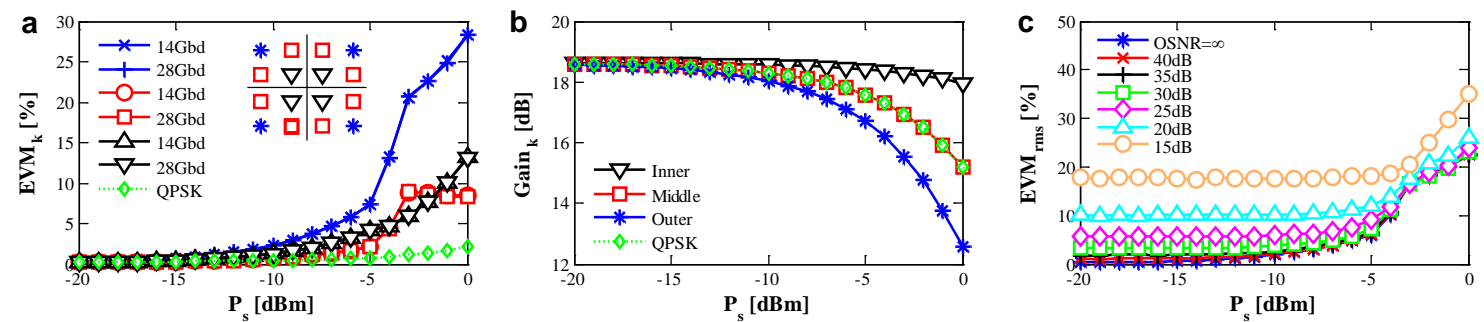

Fig. 2: Numerical results: (a) $\mathrm{EVM}_{k}$ versus average input signal power $\left(P_{s}\right)$ for 14 and 28 GBd 16-QAM and QPSK signals. The color coding refers to the position of the clusters in the constellation as shown in the inset: blue, red and black for outer, middle and inner clusters respectively; (b) cluster gain versus $P_{s}$ for 16-QAM and QPSK signal; (c) averaged EVM versus $P_{s}$ for various signal OSNRs at the FOPA input. 

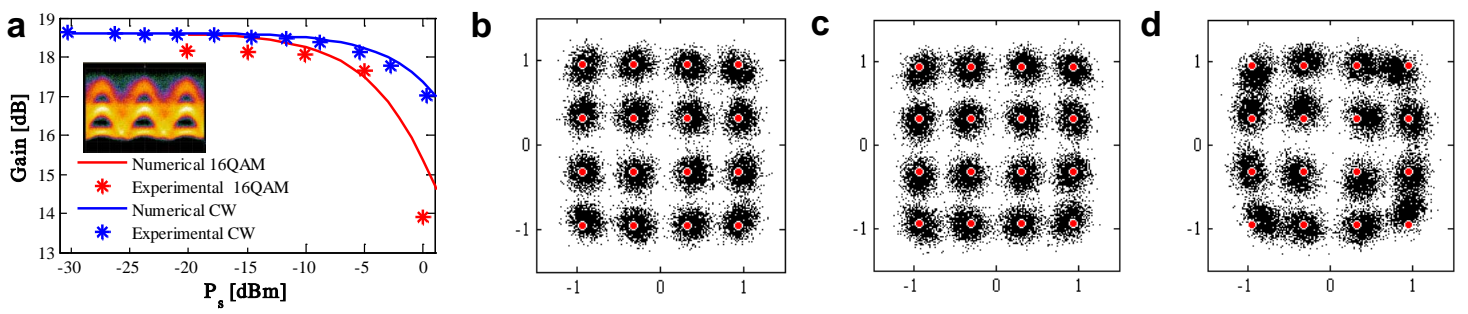

Fig. 3: Experimental results: (a) measured and simulated signal gain versus input power for CW and 16-QAM signals. The intensity eye diagram at the IQ modulator output is shown as inset; normalized constellation diagrams at FOPA input (b) and output for $P_{s}-20 \mathrm{dBm}$ (c) and $0 \mathrm{dBm}$ (d). The red dots indicate the ideal constellation.

The out-of-band noise was then suppressed with a $2.4 \cdot R_{s}$ bandwidth optical bandpass filter. Fig. 2(c) reports the averaged EVM $\left(\mathrm{EVM}_{r m s}\right)$ as a function of the signal power for various signal OSNRs. The averaged value is considered here as the cluster dependent behavior shows the same characteristics already pointed out in the noise-free analysis.

The lower the OSNR, the higher the amplitudeto-phase noise conversion and thus the higher the EVM can be expected. In the linear regime this is exactly what is observed: the output signal distortion is increased as the OSNR of the input signal decreases. When saturation effects start coming into play, the gap between the curves decreases. This suggests that the distortion induced by the saturation, i.e. the gain difference combined with the relative nonlinear phase rotation between the clusters, dominates over the noise conversion. Furthermore, the thresholds at which the saturation effects become dominant are moved towards higher power when the OSNR is decreased.

\section{Experimental results}

The signal distortion has also been evaluated experimentally by amplifying a non-return-to-zero (NRZ) 14 GBd 16-QAM signal through the same setup as the one represented in Fig. 1. Additionally, the pump linewidth was broadened by phase modulation using three tones in the $90 \mathrm{MHz}-$ $1 \mathrm{GHz}$ range, thereby shifting the stimulated Brillouin threshold of the HNLF from around $18 \mathrm{dBm}$ to over $30 \mathrm{dBm}$. The output signal was detected with a standard pre-amplified coherent receiver and the offline processing consisted of clock recovery, equalization and carrier recovery.

A comparison between the calculated and measured gain is shown in Fig. 3(a) for both $\mathrm{CW}$ and 16-QAM signals. In both cases good agreement is shown.

Fig. 3(b) reports the normalized constellation diagram at the input of the FOPA while Fig. 3(c) and (d) show the output constellations for an input power of -20 and $0 \mathrm{dBm}$, i.e. when the FOPA is operated in the linear and saturated regime, respectively. When operating the FOPA in the linear regime no additional distortion can be seen when comparing the constellation diagrams at the input and the output. In both cases the clusters are centered around the ideal constellation points. BER performances similar to ${ }^{4}$ are thus expected even with more than $18 \mathrm{~dB}$ of gain.

As the amplifier is operated in saturation, the distortion becomes evident. Comparing the demodulated constellation with the ideal constellation, it can be seen that the outer clusters are shifted to lower power while the inner cluster are moved in the opposite direction. The experiments thus show the same power squeezing characteristic highlighted in the numerical analysis.

\section{Conclusions}

The impact of saturation on the parametric amplification of 16-QAM signals has been analyzed numerically and experimentally. The EVM metric was used to numerically quantify the distortion. The respective role of the main detrimental effects, the gain difference and the nonlinear phase rotation experienced by the symbols according to their power level, has been clarified. The interplay between amplitude-to-phase noise conversion and saturation effects has been highlighted and the numerical results have been compared with experimental measurements, showing the same power squeezing effect.

\section{References}

1 P. J. Winzer et al., J. Lightwave Technol., 28, 547 (2010).

2 T. Torounidis et al., IEEE Photon. Technol. Lett., 19, 650 (2007).

3 R. Elschner et al., Proc. OFC'10, OThA4 (2010).

4 R. Elschner et al., Proc. OFC'11, OThC2 (2011).

5 I. Fatadin et al., IEEE Photon. Technol. Lett., 22, 631 (2010).

6 R. Schmogrow et al., IEEE Photon. Technol. Lett., 24, 61 (2012). 